Muxworthy, A R, Williams, W, Distribution anisotropy: the influence of magnetic interactions on the anisotropy of magnetic remanence, In: Martín-Hernández, F, Lüneburg, C M, Aubourg, C and Jackson, M,, editor, Magnetic Fabric: Methods and Applications, London, Geological Society, 2004, Pages: 37 - 4

\title{
Distribution anisotropy: the influence of magnetic interactions on the
} anisotropy of magnetic remanence.

Adrian R. Muxworthy and Wyn Williams

Grant Institute of Earth Science, University of Edinburgh, Kings Buildings, West Mains Road, Edinburgh,

EH9 3JW, UK. (e-mail: adrian.muxworthy@ed.ac.uk)

Number of words (main text and abstract) $=4437$

Number of references $=24$

Number of tables $=0$

Number of figures $=10$

Running title:

AMR and distribution anisotropy 
Abstract: The anisotropy of magnetic remanence (AMR) is often used as a tool for examining magnetic anisotropy of rocks. However, the influence of magnetostatic interactions on AMR has not been previously rigorously addressed either theoretically or experimentally, though it is widely thought to be highly significant. Using a three-dimensional micromagnetic algorithm, we have conducted a systematic numerical study of the role of magnetostatic interactions on AMR. We have considered both lineation and foliation, by modelling assemblages of ideal single domain grains and magnetically non-uniform magnetite-like cubic grains. We show that magnetostatic interactions strongly affect the measured AMR signal. It is found that depending on the orientation of the single-grain anisotropy and grain spacing it is possible for the AMR signal from a chain or grid of grains to be either oblate or prolate. For non-uniform grains, the degree of anisotropy generally increases with increasing interactions. In the modelling of AMR anisotropy, saturation isothermal remanence was chosen for numerical tractability. The influence of interactions on other types of more commonly measured AMR, are considered in light of the results in this paper. 
Anisotropy of magnetic remanence (AMR) is commonly used as an alternative to anisotropy of low-field magnetic susceptibility (AMS) as a method of determining the magnetic fabric of a rock sample. Measuring AMR has some distinct advantages over AMS, for example, if the anisotropy of partial anhysteretic remanence (AARM) is measured, then the anisotropy of different fractions of the coercivity spectrum can be assessed (Jackson et al., 1988; Aubourg \& Robion, 2002).

However, because AMR is still very much the junior partner of AMS, some theoretical aspects of AMR have not been rigorously tackled. In particular the role of distribution (or textural) anisotropy. Distribution anisotropy occurs when magnetic grains are both unevenly distributed and are close enough to interact magnetostatically, producing an asymmetric magnetic interaction field which contributes to the bulk magnetic anisotropy (Hargraves et al., 1991).

There have been several theoretical models, which have examined distribution anisotropy's contribution to AMS (Stephenson, 1994; Cañon-Tapia, 1996). These models have shown that when grains become closer and magnetostatically interact, the distributions of grains rather than their individual orientations dominate AMS. Differences between these model predictions and experimental results (Gregoire et al., 1995; Gregoire et al., 1998), have recently been attributed by Cañon-Tapia (2001) to over simplifications in the previous models.

The influence of interactions on AMR can not be directly inferred from the AMS behaviour and models, due to numerous theoretical reasons (Jackson, 1991). In this paper we investigate for the first time using a rigorous numerical model, the influence of magnetostatic interactions on AMR in assemblages of ideal single domain (SD) and pseudo-SD (PSD) magnetite-like grains, i.e., grains which display non-uniform internal magnetic structures. We consider both lineation and foliation for 
assemblages of grains with a range of both interaction spacing and single-grain anisotropy.

\section{A numerical model for distribution anisotropy}

One of the main difficulties in modelling interacting assemblages is that it is a highly non-linear problem, unlike non-interacting uniform SD grains which can be very well explained by analytical theories (e.g., Stoner \& Wohlfarth, 1948). With the rapid advancement in computing power, it has become possible to directly model this nonlinear behaviour by implementing Brown's (1963) micromagnetic formalism to study non-uniform magnetic phenomena (e.g., Williams \& Dunlop, 1989; Muxworthy et al., 2003).

The micromagnetic algorithm

In this study we have implemented a new micromagnetic algorithm. This new algorithm differs to the algorithm used in previous micromagnetic studies conducted by the Edinburgh group (e.g., Wright et al., 1997; Muxworthy et al., 2003). The algorithm is a combination of both a minimum energy conjugate-gradient (CG) algorithm (as used in previous studies) and a dynamic algorithm which follows the torque of a magnetic moment according to the Landau-Lifshitz-Gilbert (LLG) equation (Suess et al., 2002). The reasoning behind this approach is that the dynamic algorithm gives the more rigorous solution since the magnetisation between stable states must follow a physically reasonable path dictated by the LLG equation of motion, however, it is relatively slow compared to the CG method. In this combination algorithm, we use the CG algorithm to rapidly generate an initial guess for the magnetic structure, which is then put into the dynamic solver. This increases 
the efficiency of the algorithm by roughly an order of magnitude compared to the dynamic solver alone.

For the case of ideal SD assemblages each grain is represented by a simple cube, that is, each cube represents the averaged magnetisation direction of many hundreds of atomic magnetic dipole moments, or simply each cube is an ideal SD grain. The orientation of each magnetic grain can vary in direction.

The grain assemblage structure is initially calculated with the CG algorithm by minimising the total magnetic energy $E_{\text {tot, }}$ which is the sum of magnetostatic energy $E_{d}$ and the anisotropy $E_{\text {anis }}$ (Brown, 1963). $E_{\text {tot }}$ is calculated using fast-Fourier transforms (FFT), to give a local energy minimum (LEM) for the assemblage. The calculation of the energy terms and the implementation of the FFT are exactly the same as in the work of Wright et al. (1997).

After the LEM state has been estimated, the structure is optimised using the LLG algorithm. In effect instead of minimising the total energy, the LLG solver minimises the torque on each magnetic moment by calculating the total effective field. Since the LLG solver ensures that the magnetisation path between stable states is physically realistic the method is less susceptible to becoming trapped in a "false" LEM state. Additionally the LLG convergence criterion is more rigorous since it examines the torque on each magnetisation vector rather than simply the gradient of the total energy.

For the PSD models, to model the non-uniform internal structures each grain is represented by $7 \times 7 \times 7$ cubes or cells compared to 1 in the ideal SD case, and the 
exchange interaction within each grain accommodated, i.e., the exchange interaction between the $7 \times 7 \times 7$ cells representing each grain.

Modelling distribution anisotropy

There are several different types of magnetic remanence and consequently several different types of AMR. The most commonly examined is AARM, as it is rapidly, isothermally induced and it is possible to access distinct fractions of the coercivity distribution. However, AARM is practically impossible to model using a micromagnetic approach due to the difficulty in simulating the alternating field. Instead we have considered the anisotropy of isothermal remanence (AIRM), which is utilised when the samples are too weak to consider using AARM (Stephenson et al., 1986; Potter \& Stephenson, 1988). As a first approach to resolve the initial starting-state problem which exists for non-saturating IRM models, we have considered anisotropy of saturation IRM (ASIRM).

For linear anisotropic magnetisations like AARM or thermoremanent AMR the directional variability is mathematically described by a second-rank symmetric tensor (Tarling \& Hrouda, 1993). This tensor can be represented geometrically as a triaxial ellipsoid, with the principal axes parallel to the eigenvectors of the second-rank tensor and with the principal axis lengths equal to the corresponding eigenvalues of the tensor. However, for strong field remanences like SIRM, the relationship between the magnetisation and field is non-linear meaning that the magnetisation and the field strength are not related by a second-rank tensor. It might be argued then, that modelling ASIRM is not the best choice of remanences to consider, however, from a computational point of view the other types are impractical. 
The distribution anisotropy is treated in a similar manner to that reported in similar AMS studies (e.g., Stephenson, 1994; Cañon-Tapia, 1996; Gregoire et al., 1998). We consider both lineation (chains) and foliation (planes/grids) in assemblages of ideal SD and PSD grains.

For the SD models we considered three single-grain anisotropy (SGA) distributions: "aligned" (AR) and "side-by-side" (SBSR) regimes (Fig. 1), as well as randomly distributed SGA regimes (RR). The AR and SBSR configurations are the extreme cases and were considered in previous AMS models (Stephenson, 1994; CañonTapia, 1996, 2001). For the PSD models only AR and SBSR were considered.

To conform with previous anisotropy studies we consider uniaxial anisotropy. Most previous studies have used elongation ratios to describe the uniaxial anisotropy in their models (e.g., Stephenson, 1994; Cañon-Tapia, 1996, 2001). In order to maintain the computational efficiencies of the FFT used in the CG algorithm, rather than varying the elongation ratio we added an additional energy term of the form $E_{\text {anis }}$ $=K_{U} \sin ^{2} \theta$, where $\theta$ is the angle between the elongation axis and the magnetisation and $K_{U}$ is a parameter related to the elongation ratio $q$ of the grain. The value of $K_{U}$ is determined by using the standard formula $K_{U}=\mu_{0} M_{S}^{2} N(q) / 2$, where $\mu_{0}$ is the permeability of free space, $M_{S}$ the spontaneous magnetisation and $N(q)$ is the demagnetising factor which is simply a function of $q$ (Nagata, 1961).

\section{Distribution anisotropy and ideal uniaxial SD grains}

Treating SD grains as perfectly uniformly magnetised particles has been shown to not significantly alter their interaction behaviour (Muxworthy et al., 2003), and allows large particle assemblages to be modelled 
Lineation for uniform SD grain assemblages

Lineation due to distribution anisotropy is where the interaction field is primarily in one direction only (Tarling \& Hrouda, 1993). Such interaction fields are often modelled as simple chain of grains (Stephenson, 1994; Cañon-Tapia, 1996). We have modelled ASIRM for chains of 25 identical grains with identical interaction spacing. It has been shown experimentally that chain lengths of $\sim 10$ particles are as a first approximation sufficient to represent infinite chains (Fuller, 1961). For AR, the magnetostatic interaction field $\left(H_{1}\right)$ and the SGA field $\left(H_{\mathrm{SGA}}\right)$ are parallel, whereas for SBSR $H_{1}$ and $H_{\mathrm{SGA}}$ are perpendicular (Fig. 1). $H_{1}$ is in the direction of the chain.

Due to symmetry, when calculating ASIRM for AR only two field directions, i.e., $F_{x}$ and $F_{y}$, were determined as ASIRM in the $y$ and $z$ directions, i.e., ASIRM $_{A R y}$ and $\mathrm{ASIRM}_{\mathrm{ARz}}$, are identical (Fig 1). The remanence tensor is calculated by simulating a SIRM in three perpendicular directions and measuring the intensity of the component of remanence that is acquired parallel to the applied field.

For $A_{S I R M}$ ARx,$A_{S I R M}$ ARy and $A_{S I R M}$ ARz the magnetic moments are aligned in the direction parallel to $x$, regardless of interaction spacing $d / r$ or $q$ (Fig. 2a). Both $H_{1}$ and $H_{\mathrm{SGA}}$ are parallel and have the same effect, i.e., rotating the magnetic moments into the $x$ direction. For fields applied in the $y$ - and $z$ - directions, i.e., $F_{y}$ and $F_{z}$, the particles rotate into the $x$-direction as the field is decreased from saturation. The shape parameter $T$ is equal to -1 for this highly anisotropic configuration. $T$ is given by (Jelínek, 1981),

$$
T=\left[\frac{2 \ln \left(K_{2} / K_{3}\right)}{\ln \left(K_{1} / K_{3}\right)}\right]-1
$$


where $K_{1}, K_{2}$ and $K_{3}$ are the maximum, intermediate and minimum values of SIRM for the three calculated directions. If $-1<T<0$, then the system shows lineation (prolate), whereas, if $0<T<1$ then the sample displays foliation (oblate) (Jelínek, 1981; Hrouda, 1982).

In contrast to the AR case, the SBSR is strongly influenced by interactions (Fig. 2b); $H_{1}$ and $H_{\mathrm{SGA}}$ are perpendicular. At large interaction spacing (approaching the true non-interacting state), i.e., $d / r \rightarrow 0$, SGA dominates, and ASIRM is close to 1 for $F_{z}$ (ASIRM $M_{S B S Z}$ ), and zero for $F_{y}$ and $F_{x}$. However, as the interactions start to dominate

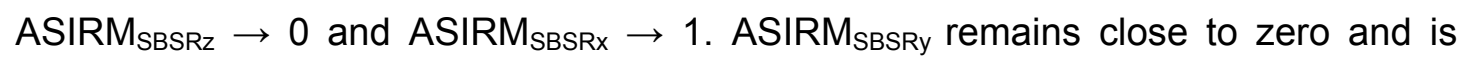
invariant to $d / r$. The interaction spacing at which $H_{1}$ starts to dominate depends on the size of $K_{\mathrm{u}}$, which is proportional to the size of the anisotropy effective field. The larger $q$ the less the magnetic structure is affected by the interaction field. We have not considered the extreme situation where $K_{\mathrm{u}}=0$, however, in this case for ideal SD grains the interaction field would control the behaviour even at $d / r=0$.

For non-interacting particles, i.e., $\mathrm{d} / \mathrm{r}=0$, the linear $H_{\mathrm{SGA}}$ dominates the magnetic signal and $T=-1$ (Fig. 3). For strongly interacting systems, i.e., $d / r \geq 0.8$, then the linear $H_{1}$ dominates and $T$ is also equal to -1 . However, for intermediate, states, $H_{1}$ and $H_{\mathrm{SGA}}$ are in competition with each other, and depending on the balance of the energies, it is possible for $T>0$, i.e., an oblate signal. This is seen for $q=1.1$ (Fig. 3).

The behaviour of the randomly distributed anisotropy regime (RR) is markedly different, primarily because $H_{\mathrm{SGA}}$ has no preferred direction. For large interaction spacings, i.e., $\mathrm{d} / \mathrm{r} \rightarrow 0, \mathrm{ASIRM} \mathrm{RR}_{\mathrm{R}}$ approaches $\sim 0.5$, i.e., the expected $M_{\mathrm{RS}} / M_{\mathrm{S}}$ value for randomly distributed non-interacting SD grains (Dunlop \& Özdemir, 1997). As the 
assemblage is only for 25 grains the small differences between 0.5 and the calculated values can be attributed to the low number of grains not giving a true random distribution. As $\mathrm{d} / \mathrm{r} \rightarrow 1$, the moments align in the direction of $H_{1}$, i.e., $A S I R M_{R R x} \rightarrow 1$ and $A S I R M_{R R y}$ and $A_{S I R M} M_{R z} \rightarrow 0$. Note $A_{S I R M_{R R y}}$ and $A S I R M_{R R z}$ are symmetric as in the AR case.

Again there is a dependency on $q$. The smaller $q$, the more the particles are influenced by $H_{1}$. The shape parameter $T$ is -1 for all values of $d / r>0$. A random assemblage at $\mathrm{d} / \mathrm{r}=0$ is isotropic, for which $T$ is not defined.

For the random regime the Jelínek's (1981) corrected anisotropy degree $P_{J}$ (sphere to ellipsoid; $1 \rightarrow \infty$ ) is plotted as a function of $d / r$ in Fig. 4 . Clearly the degree of anisotropy increases as the $d / r$ increases. This effect is most pronounced for $q=1.1$.

\section{Foliation for uniform SD grain assemblages}

In a similar set of simulations, foliation was examined by simulating ASIRM for grids of $10 \times 10$ arrays of SD particles. In the foliation model the direction of the $H_{1}$ is more localised and variable than in the lineation system; it lies within the $x-y$ plane rather than along an axis. AR is now for anisotropy aligned in either the $x$ or $y$ directions, and SGSR is for SGA out of the $x-y$ plane, i.e., in the $z$ direction (Fig. 5).

Generally the effect of interactions is to decrease ASIRM for anisotropies parallel to the field, and to cause small increases in ASIRM for anisotropies perpendicular to the fields (Fig 6a and 6b), e.g., $A_{S I R M_{S B S R z}}$ decreases from 1 to 0 with increasing interactions, whilst $A S I R M_{S B S R x}$ and $A S I R M_{S B S R y}$ increase slightly from 0 . In the AR model the $H_{1}$ and $H_{\mathrm{SGA}}$ are within the same plane. The effect of interactions is more pronounced in SBSR model, where $H_{1}$ rotates the moments into the $x-y$ plane, i.e., 
the hard uniaxial SGA plane (Fig 6b). Similarly to the lineation models, the effect of interactions is highly dependent on $q$. For $q=1.1$, ASIRM $_{\text {ARy }}$ increases slightly as $\mathrm{d} / \mathrm{r}$ increases from 0.8 to 1 . This increase is repeatable, and is due to the structure being unable to break the symmetry for large $H_{1}$.

The behaviour of the random assemblage is markedly different (Fig. 6c). For small $\mathrm{d} / \mathrm{r}$, ASIRM approach $\sim 0.5$ for $\mathrm{F}_{x}, \mathrm{~F}_{y}$ and $\mathrm{F}_{z}$ as expected for randomly distributed non-interacting uniaxial SD particles. For the out-of-plane field $F_{z}$, the effect of interactions is to decrease $A S I R M_{R R z}$. $A S I R M_{R R z} \rightarrow 0$ as $d / r \rightarrow 1$. Interactions initially increase both $A S I R M_{R R x}$ and $A S I R M_{R R y}$ as $d / r$ increases, due to the rotation of the moments into the $x-y$ plane. As $\mathrm{d} / \mathrm{r} \rightarrow 1 \sim 0$, both ASIRM $_{\mathrm{RRx}}$ and ASIRM $_{\mathrm{RRy}}$ then decrease to zero as $d / r \rightarrow 1 \sim 0$, giving rise to a peak in $A_{S I R M}$ RRx and $A S I R M_{R R y}$ at intermediate values of $d / r$. These peaks are clearly dependent on $q$. This decrease in $A_{S I R M}$ RRx and $A S I R M_{R R y}$ as $d / r \rightarrow 1$, is due to the rearrangement of the moments in the $x-y$ plane as they respond to the magnetostatic interaction field. As $d / r \rightarrow 1$ some moments will lie parallel and others anti-parallel to the applied field direction in the $x-y$ plane, giving a net magnetisation of $\sim 0$. This contrast sharply with models of susceptibility where in-plane and mean susceptibilities continue to increase (CañonTapia, 1996). For the susceptibility model of Cañon-Tapia (1996), the effect of interactions was to decrease the micro-coercivity of each grain, which in turn lead to an increase in the susceptibility. This difference between remanence and susceptibility is due to their intrinsically different nature; one is a zero-field state and the other an in-field state.

As $d / r$ increases, the more planar $H_{1}$ starts to dominate over the more linear $H_{\text {SGA. }}$. Consequently for AR and SBSR $T$ switches from negative to positive, i.e., from a prolate to an oblate signal (Figs. $6 \mathrm{~d}$ and $6 \mathrm{e}$ ). For $\mathrm{RR}, T=1$ for all $\mathrm{d} / \mathrm{r}>0$ due to $H_{\mathrm{SGA}}$ being isotropic and $H_{1}$ having high-planar symmetry (Fig. 6f). A random assemblage 
at $d / r=0$ is isotropic, for which $T$ (equation (1)) is not defined, though it could be argued that it should take the value zero.

For $\mathrm{RR}$ with $q=1.1, P_{\mathrm{J}}$ exhibits a peak at $\mathrm{d} / \mathrm{r} \sim 0.6$ (Fig. 7$)$. This peak is caused by the weak SGA field being unable to break the symmetry of the magnetic structure as $H_{\mathrm{EXT}}$ decreases, resulting in large values of $A S I R M_{R R x}$ and $A_{S I R M_{R R y}}$ and a peak in $P_{\mathrm{J}} . \mathrm{RR}$ with $q=1.3$ and 1.5 displays the same gradual increase with increasing $\mathrm{d} / \mathrm{r}$ as in the lineation model (Fig. 4). The magnitude of $P_{\mathrm{J}}$ is less.

\section{Distribution anisotropy and non-uniform magnetic structures}

In the previous section it was assumed that each particle has a uniform internal magnetic structure. This assumption is valid for very small grains, however, larger grains of soft magnetic minerals like magnetite have complicated non-uniform magnetic structures. As the SD to multidomain (MD) or PSD grain size threshold $d_{0}$ is approached, SD grains display non-uniform flower structures (Fig 8a), whilst in magnetite the smallest MD grains display vortex structures (Fig 8b). The magnetic behaviour of these vortex structures is significantly different to SD grains (Williams \& Dunlop, 1989). We investigate their AMR here.

The size of a non-uniform grain is critical to its magnetic behaviour. We model only one size of grain; cubic grains with a length of $90 \mathrm{~nm}$. This size was carefully selected, because it is known that the magnetic structure of grains just above $d_{0}$ are very strongly influenced by magnetostatic interactions (Muxworthy et al., 2003). For $90 \mathrm{~nm}$ grains the vortex structure is the lowest energy state in a zero-field environment. However, in the presence of small external fields (either $H_{\mathrm{EXT}}$ or $H_{1}$ ), the SD or flower state becomes energetically more favourable. Muxworthy et al. (2003) showed that magnetostatic interactions effectively increase $d_{0}$. 
Although we are modelling only the smallest MD grains, by considering the role of interactions in assemblages of such grains just above $d_{0}$, we are arguably examining MD grains, which are most strongly influenced by magnetostatic interactions.

Modelling both the internal structure as well as the interaction fields, makes the problem computationally more intensive than for uniform SD models. This significantly limits the number of grains and variations in $\mathrm{d} / \mathrm{r}$ that can be modelled. This type of computational limit is not uncommon. In previous numerical models, which assessed the role of interactions on AMS, it was commonly assumed that calculating only the magnetic signal of a grain in the middle of a short chain was sufficient to predict the behaviour of an infinite chain. This approximation is not applicable to models for ASIRM. For example, if one considers a SIRM for a $3 \times 3$ grid of grains, then for certain interaction spacings and field orientations, the magnetic moment of the middle grain is reversed in direction with respect to the applied field, i.e., a negative SIRM, yet the entire assemblage has a positive SIRM. Clearly, using the behaviour of the middle grain to predict the behaviour of a larger assemblage is not valid for SIRM models.

For our lineation model, chains of 15 grains were modelled, whilst for foliation model grids of $5 \times 5$ grains were considered. Due to the small number of grains within the models only AR and SBSR are considered.

Each grain is modelled with a resolution of $7 \times 7 \times 7$ cells. This is a little lower than that predicted to be the minimum to model magnetite-like cubes $90 \mathrm{~nm}$ in size, i.e., $13 \times 13 \times 13$ (Rave et al., 1998). By implementing a slightly lower resolution, we potentially underestimate the exchange energy, however, as we are primarily 
interested in changes with interaction spacing then our model will still show the same trends.

\section{Lineation of non-uniform magnetic structures}

The internal magnetic structures and consequent SIRM values of the grains within the chains are strongly influenced by interactions. For example, consider the normalised ASIRM $_{\mathrm{AR}}$ for $q=1.1$ : $\mathrm{ASIRM}_{\mathrm{ARx}}=0.17$ for $\mathrm{d} / \mathrm{r}=0.5$, but for $\mathrm{d} / \mathrm{r}=0.875$, $\mathrm{ASIRM}_{\mathrm{ARx}}=0.95$. This is because for $\mathrm{d} / \mathrm{r}=0.5$ all the particles are in a vortex state, which has a lower remanence, but as the interactions increase the particles reside in a flower structure (Fig 9a); $H_{1}$ increases $d_{0}$ to $>90 \mathrm{~nm}$, and ASIRM $_{\mathrm{ARx}}$ similarly increases. Similar effects are seen in the domain structures of all the models.

For non-interacting particles, i.e., $\mathrm{d} / \mathrm{r}=0$, the linear $H_{\mathrm{SGA}}$ dominates the magnetic signal and $T=-1$ (Fig. 10a). For strongly interacting systems, i.e., $d / r \geq 0.875$, then the linear $H_{1}$ dominates and $T$ is also equal to -1 . However, for intermediate, states, for the SBSR $H_{1}$ and $H_{\mathrm{SGA}}$ are in competition with each other, and it is possible for $T>$ 0, in a similar manner as seen for the SBSR SD particles (Fig. 3). This is seen for both $q=1.1$ and 1.3 (Fig. 10a).

Ideally, more points are needed in Fig. 10a, however, this problem is computationally intensive. The trends can still be seen.

\section{Foliation of non-uniform magnetic structures}

As in the uniform SD models, $H_{1}$ lies within the $x-y$ plane for the foliation scheme (Fig. 4). Similar to the lineation model, the interaction field effectively increases $d_{0}$ making the flower state more favourable than the vortex state as $\mathrm{d} / \mathrm{r} \rightarrow 1$. However, this does 
not effect the magnitude of the SIRM as it does for the lineation model since the domain state and domain orientation can change. For example, consider the zerofield state for the aligned assemblage with $q=1.3$, with the field applied in the $x$ direction, i.e., $A_{S I R M}$ ARx. For $\mathrm{d} / \mathrm{r}=0.5$ all 25 grains are in a vortex state, whereas for $\mathrm{d} / \mathrm{r}=0.875$ each grain is in a flower state, though the reduced remanences are quite similar: $A S I R M_{A R x}=0.13$ for $\mathrm{d} / \mathrm{r}=0.5$ and 0.19 for $\mathrm{d} / \mathrm{r}=0.875$.

Even though the SIRM magnitude does not change significantly, the symmetry of the system is strongly effected. As $d / r$ increases, the planar $H_{1}$ dominates over the linear $H_{\mathrm{SGA}}$, and the shape parameter switches from -1 to $>0$ (Fig. 10b). However, $\mathrm{d} / \mathrm{r}=$ 0.875 for the SBSR model, $T<0$. It is believed that this is an artefact of the small assemblage size, i.e., only 25 grains, and that for a larger assemblage size or a less symmetric distribution that $T>0$.

\section{Discussion and conclusions}

The situation for the foliation regimes is easily summarised. For systems with a linear anisotropic $H_{\mathrm{SGA}}$, then regardless of the exact anisotropy distribution as $\mathrm{d} / \mathrm{r}$ increases $T$ will increase from negative to positive values as the planar $H_{1}$ starts to dominate. The interaction spacing at which $T$ becomes positive depends on the size and orientation of $H_{\mathrm{SGA}}$ and $H_{1}$. These two fields are in direct competition with each other. If $H_{\mathrm{SGA}}$ is isotropic, i.e., a random distribution, then it is shown that for the SD assemblage for $\mathrm{d} / \mathrm{r}>0, T>0$. How would a random distribution of non-uniform grains (or PSD) grains behave? Unfortunately it was not possible to model large distributions to obtain genuine random distributions, however, it is possible to predict their behaviour qualitatively. For foliation, the behaviour is quite simple, for $d / r>0$, then $T$ would also be $>0$, due to the planar $H_{1}$. 
For the lineation models, the behaviour is a little more complicated. Assuming some linear anisotropic $H_{\mathrm{SGA}}$, then for small $\mathrm{d} / \mathrm{r}, T<-1$, similarly for large $\mathrm{d} / \mathrm{r}$, due to the linearity of $H_{1}, T<0$. However, at intermediate states depending on $\mathrm{d} / \mathrm{r}$, anisotropy, relative directions of $H_{\mathrm{SGA}}$ and $H_{1}$, it is possible to obtain values for $T>0$. That is, what would appear to be a highly linear system, can yield a foliation signal. It can be hypothesised for a random assemblage of PSD grains that $T$ would similarly always be $<0$.

For both the lineation and foliation models the size of $P_{\mathrm{J}}$ roughly increases with increasing $d / r$.

There are no direct experimental results to compare the model results to, however, Gregoire et al. (1995) measured the shape parameter for AMS for two large MD magnetite crystals. They found that for a SBS chain configuration that $T$ could switch from $T<0$, to $T>0$ and then back to $T<0$ as $\mathrm{d} / \mathrm{r}$ increased towards one, in a similar manner to that seen in Figures 3 and 9a for AMR. It is difficult to draw too strong comparisons due to the differences in grain size and between AMS and AMR.

ASIRM is not commonly measured due to the desire to measure a linear tensorial regime. A question that might be raised after reading this paper is; what can be speculated from the results here in this paper about the role of interactions on the more commonly measured AARM? As the alternating field decreases, there will be some critical point dependent on $\mathrm{d} / \mathrm{r}$, where the interaction field dominates over the alternating field, and the behaviour of the assemblage controlled by this interaction field. For highly interacting regimes, i.e., where $d / r \rightarrow 1$, then the interactions will dominate the system at large alternating fields and the results will be very similar to those for ASIRM. For weak and moderate interacting regimes, then the role of interactions is a little harder to predict, due to the non-linearity of the problem. It is 
likely that the features found for the ASIRM are less pronounced for AARM, as the symmetry seen in the highly-uniform ASIRM model structures will be broken by the alternating field resulting in the "transition" from non-interacting to interacting behaviour occurring at higher values of $\mathrm{d} / \mathrm{r}$.

The natural extension of this study, would be to model the role of interactions on the anisotropy of non-saturating IRM, and to assess the role of the initial starting state of the assemblage on the behaviour.

We thank both $\mathrm{M}$. Jackson and $\mathrm{H}$. de Wall for independently suggesting the concept of this paper, and M. Fuller, B. Moskowitz and M. Jackson for constructive reviews. This work was funded through NERC grant NER/A/S/2001/00539 to W.W.

\section{References}

Aubourg, C. \& Robion, P. 2002. Composite ferromagnetic fabrics (magnetite, greigite) measured by AMS and partial AARM in weakly strained sandstones from western Makran, Iran, Geophysical Journal International, 151, 729-737.

Brown, W.F., Jr. 1963. Micromagnetics, John Wiley, New York.

Cañon-Tapia, E. 1996. Single-grain versus distribution anisotropy: a simple three-dimensional model, Physics of the Earth and Planetary Interiors, 94, 149-158.

Cañon-Tapia, E. 2001. Factors affecting the relative importance of shape and distribution anisotropy in rocks: theory and experiments, Tectonophysics, 340, 117-131.

Dunlop, D.J. \& Özdemir, Ö. 1997. Rock Magnetism: Fundamentals and Frontiers, Cambridge University Press, Cambridge.

Fuller, M. D., 1961. Magnetic Anisotropy of Rocks, Ph.D. Thesis, University of Cambridge.

Gregoire, V., Blanquat, M.D., Nedelec, A. \& Bouchez, J.L. 1995. Shape Anisotropy Versus MagneticInteractions of Magnetite Grains - Experiments and Application to AMS in Granitic-Rocks, Geophysical Research Letters, 22, 2765-2768.

Gregoire, V., Darrozes, J., Gaillot, P., Nedelec, A. \& Launeau, P. 1998. Magnetite grain shape fabric and distribution anisotropy vs rock magnetic fabric: a three-dimensional case study, Journal of Structural Geology, 20, 937-944.

Hargraves, R.B., Johnson, D. \& Chan, C.Y. 1991. Distribution anisotropy: the cause of AMS in igneous rocks?, Geophysical Research Letters, 18, 2193-2196.

Hrouda, F. 1982. Magnetic anisotropy of rocks and its application in geology and geophysics, Geophysical Surveys, 5, 37-82.

Jackson, M. 1991. Anisotropy of magnetic remanence - a brief review of mineralogical sources, physical origins, and geological applications, and comparison with susceptibility anisotropy, Pure Applied Geophysics, 136, 1-28.

Jackson, M.J., Gruber, W., Marvin, J.A. \& Banerjee, S.K. 1988. Partial anhysteretic remanence and its anisotropy: applications and grainsize dependence, Geophysical Research Letters, 15, 440-443.

Jelínek, V. 1981. Characterization of the magnetic fabrics of rocks, Tectonophysics, 79, T63-T67.

Muxworthy, A.R., Williams, W. \& Virdee, D. 2003. The effect of magnetostatic interactions on the hysteresis parameters of single-domain and pseudo-single domain grains, Journal of Geophysical Research-Solid Earth, 108, B11, 2517, doi:10.1029/2003JB002588.

Nagata, T. 1961. Rock Magnetism., revised edition, Mazuren Company Ltd, Tokyo.

Potter, D.K. \& Stephenson, A. 1988. Single-domain particles in rocks and magnetic fabric analysis, Geophysical Research Letters, 15, 1097-1100. 
Rave, W., Fabian, K. \& Hubert, A. 1998. The magnetic states of small cubic magnetic particles with uniaxial anisotropy, Journal of Magnetism and Magnetic Materials, 190, 332-348.

Stephenson, A., 1994. Distribution anisotropy: two simple models for magnetic lineation and foliation, Physics of the Earth and Planetary Interiors, 82, 49-53.

Stephenson, A., Sadikun, S. \& Potter, D.K. 1986. A theoretical and experimental comparison of the anisotropies of magnetic susceptibility and remanence in rocks and minerals, Geophysical Journal of the Royal Astronomical Society, 84, 185-200.

Stoner, E.C. \& Wohlfarth, E.P. 1948. A mechanism of magnetic hysteresis in heterogeneous alloys, Philosophical Transactions of the Royal Society of London, Series A, 240, 599-602.

Suess, D., Tsiantos, V., Schrefl, T., Fidler, J., Scholz, W., Forster, H., Dittrich, R. \& Miles, J. 2002. Time resolved micromagnetics using a preconditioned time integration method, Journal of Magnetism and Magnetic Materials, 248, 298-311.

Tarling, D.H. \& Hrouda, F. 1993. The Magnetic Anisotropy of Rocks, Chapman \& Hall, London.

Williams, W. \& Dunlop, D.J. 1989. Three-dimensional micromagnetic modelling of ferromagnetic domain structure, Nature, 337, 634-637.

Wright, T.M., Williams, W. \& Dunlop, D.J. 1997. An improved algorithm for micromagnetics, Journal of Geophysical Research, 102, 12085-12094. 


\section{Figure Captions.}

Figure 1. Schematic showing the lineation (chain) arrangement in the model. Two single-grain anisotropy distributions are shown: AR and SBSR. Each grain has a uniaxial anisotropy. For AR the anisotropy is in the same direction as the chain, for SBSR the anisotropy direction is perpendicular to the chain direction. Random anisotropy distributions were also modelled, though a schematic of this is not depicted. Only part of the chain is shown.

Figure 2. SIRM versus $\mathrm{d} / \mathrm{r}$ for chains of ideal SD grains with three different singlegrain anisotropy distributions (a) AR, (b) SBSR and (c) RR. Three single-grain anisotropies are shown on each diagram; these are related to the elongation ratio $q$. $q=1.1$ has the smallest anisotropy, and $q=1.5$ largest. For each single-grain anisotropy distribution, SIRM was calculated for a field simulated in the $x, y$, and $z$ directions, i.e., $F_{\mathrm{x}}, F_{\mathrm{y}}$ and $F_{\mathrm{z}}$. Due to symmetry considerations, some field directions were identical. For simplicity and clarity in (b) the $F_{\mathrm{y}}$ data is not shown; it equalled $\sim 0$ for all $d / r$. When $d / r=0$ the grains are non-interacting, and when $d / r=1$ the particles are physically touching.

Figure 3. Shape parameter $T$ versus $\mathrm{d} / \mathrm{r}$ for the SD SBSR lineation (chain) model. Three single-grain anisotropies are shown on each diagram, i.e., $q=1.1,1.3$ and 1.5. When $T<0$, the assemblage has a prolate signature, and when $T>0$ an oblate one. The aligned and random models both gave a shape parameter of $T=-1$ for all $\mathrm{d} / \mathrm{r}$.

Figure 4. Degree of anisotropy $P_{\mathrm{J}}$ for a random assemblage of uniform SD grains in a chain (lineation). Three single-grain anisotropies are shown on each diagram; these are related to the elongation ratio $q . q=1.1$ has the smallest anisotropy, and $q=1.5$ largest. 
Figure 5. Schematic showing the foliation (grid) arrangement in the model. Two single-grain anisotropy distributions are shown: AR and SBSR. For AR the anisotropy is in the same plane as the grain distribution, for SBSR the anisotropy direction is perpendicular to this plane. Random anisotropy distributions were also modelled, though a schematic of this is not depicted. Only part of the grid is shown.

Figure 6. SIRM versus $\mathrm{d} / \mathrm{r}$ for grids (foliation) of ideal SD grains with three different single-grain anisotropy distributions (a) AR, (b) SBSR and (c) RR, and the corresponding shape-parameter $T$ versus $d / r$ for (d) AR, (e) SBSR and (f) RR. Three single-grain anisotropies are shown on each diagram, i.e., $q=1.1,1.3$ and 1.5. For each single-grain anisotropy distribution, SIRM was calculated for a field simulated in the $\mathrm{x}, \mathrm{y}$, and $\mathrm{z}$ directions, i.e., $F_{\mathrm{x}}, F_{\mathrm{y}}$ and $F_{\mathrm{z}}$. Due to symmetry considerations, some field directions were identical.

Figure 7. Degree of anisotropy $P_{\mathrm{J}}$ for a random assemblage of uniform SD grains in a grid pattern (foliation). Three single-grain anisotropies are shown on each diagram; these are related to the elongation ratio $q . q=1.1$ has the smallest anisotropy, and $q$ $=1.5$ largest.

Figure 8. Domains states occurring in cubic grains of magnetite-like minerals at room temperature for a grain with edge length of $90 \mathrm{~nm}$ (a) single domain (flower state) and (b) single vortex state. In this paper the term "SD state" refers not just to homogeneous magnetisation structures as in Néel theory, but also to non-uniform domain structures as shown in (a) which are basically SD-like with a degree of flowering towards the edges of the grain. 
Figure 9. Two middle-segments from the lineation model, showing the contribution of magnetostatic interactions to remanence domain structures of an aligned regime. The upper chain has a closer interaction spacing $(d / r=0.875)$, the lower a larger spacing and $d / r=0.5$. For $d / r=0.875$ the grains are clearly in the flower state (Fig. 7a) and for $d / r=0.5$ the grains are all in a vortex state (Fig. $7 b$ ). The effect of interactions is to increase $d_{0}$. The direction of the applied field $\left(F_{\mathrm{x}}\right)$ and the singlegrain anisotropy field (AR) are also highlighted. The grains depicted are grains 7, 8 and 9 from a chain of 15 .

Figure 10. Shape parameter $T$ versus $\mathrm{d} / \mathrm{r}$ for the PSD models: (a) lineation (chain) and (b) foliation model. Two single grain anisotropies are shown ( $q=1.1$ and 1.3). For the SBSR model for $\mathrm{d} / \mathrm{r}=0.875 T<0$. It is believed that this is an artefact of the small assemblage size, and for larger assemblage models $T$ would be larger $>0$. 


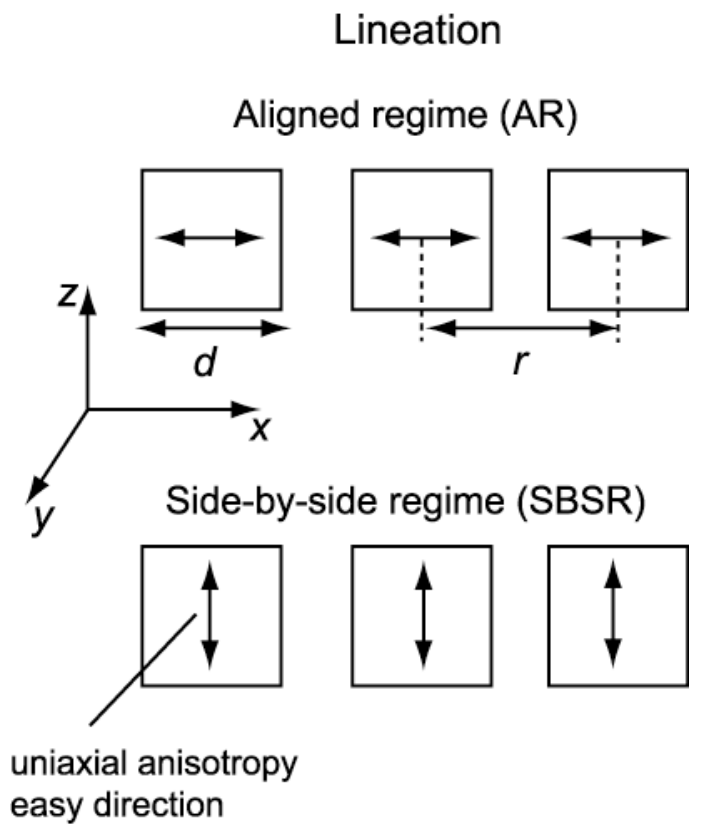

Figure 1. 


\section{Lineation}
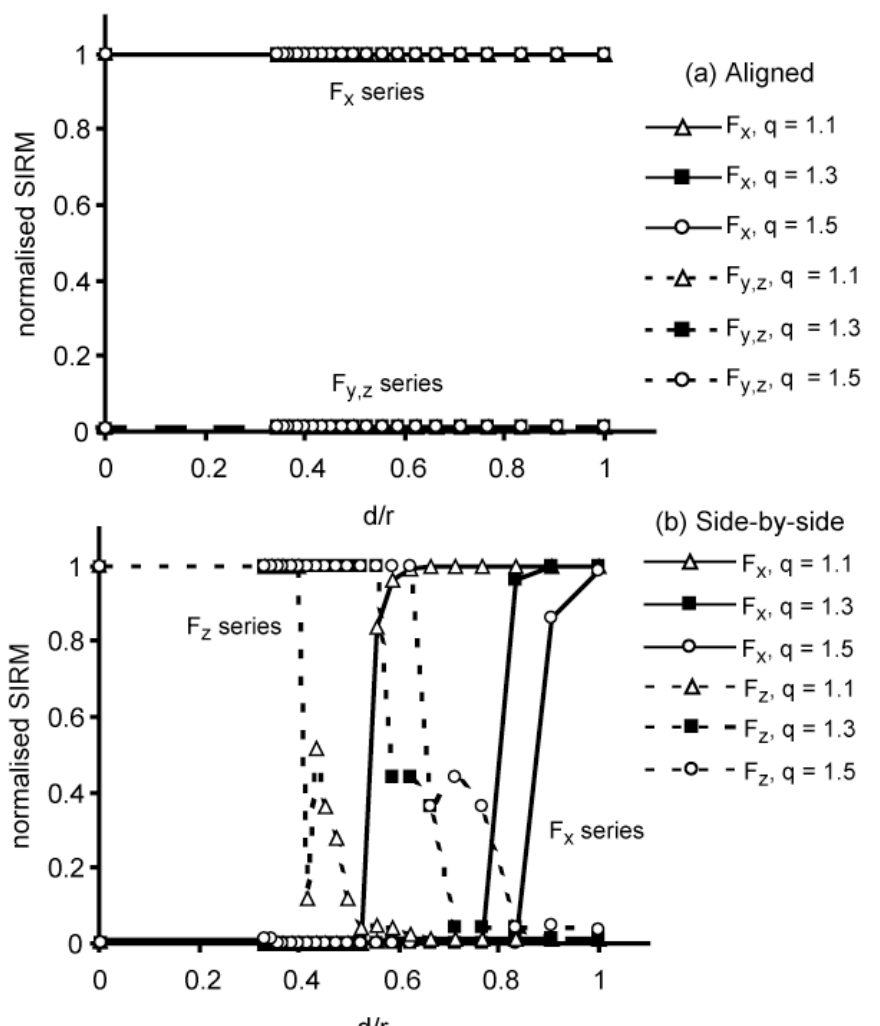

(b) Side-by-side

$\triangle-F_{x}, q=1.1$

$\longrightarrow F_{x}, q=1.3$

$\longrightarrow F_{x}, q=1.5$

- $\Delta-\quad-F_{z}, q=1.1$

- $-F_{z}, q=1.3$

-o- $F_{z}, q=1.5$

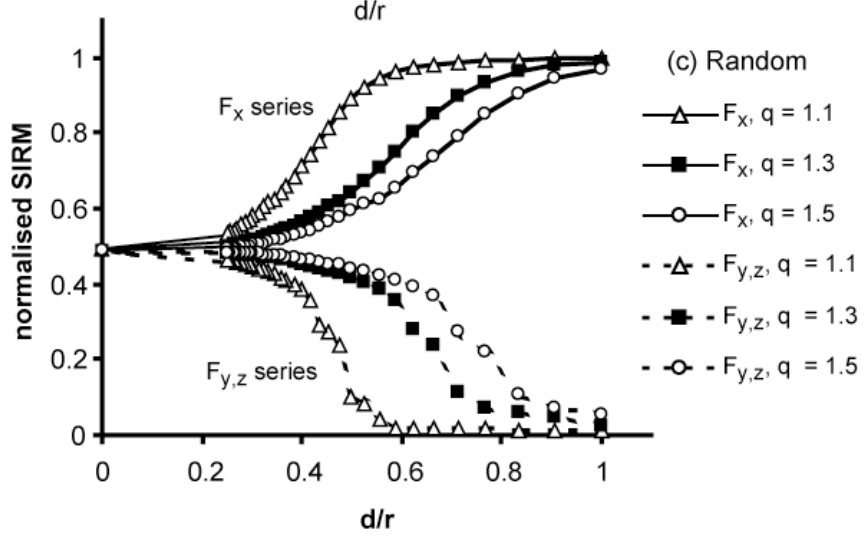

Figure 2. 


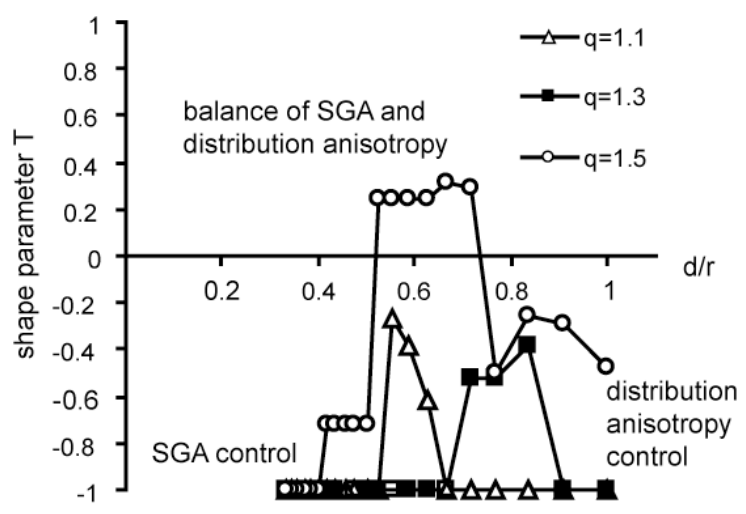

Figure 3.

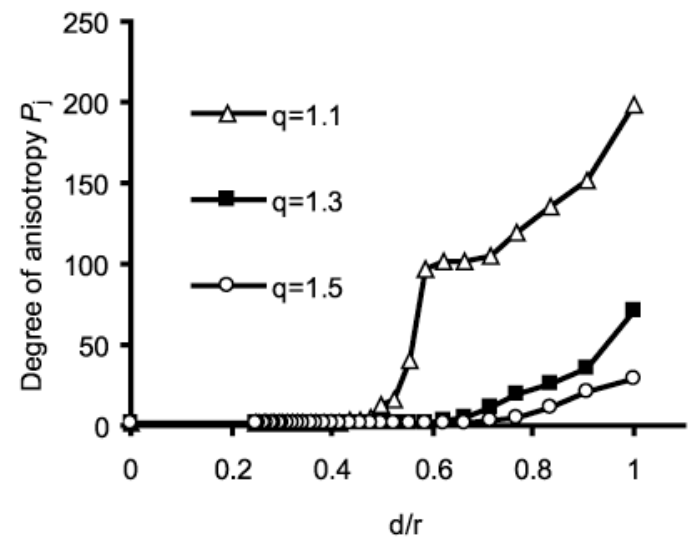

Figure 4. 
Foliation

Aligned regime (AR)
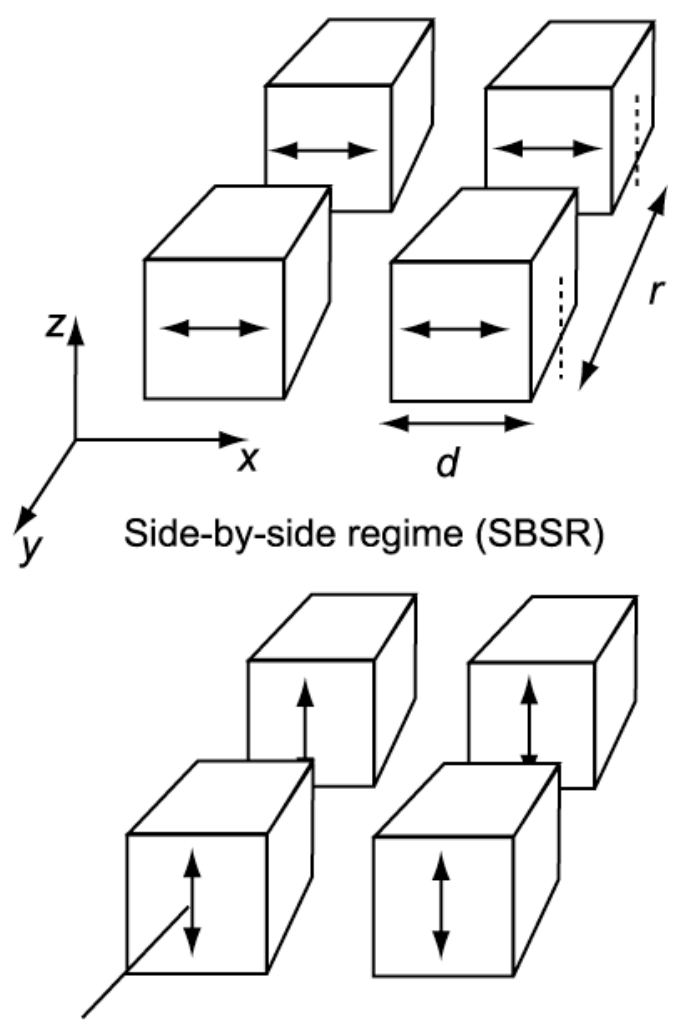

uniaxial anisotropy

easy direction

Figure 5. 
Foliation

SIRM

Aligned shape parameter $T$
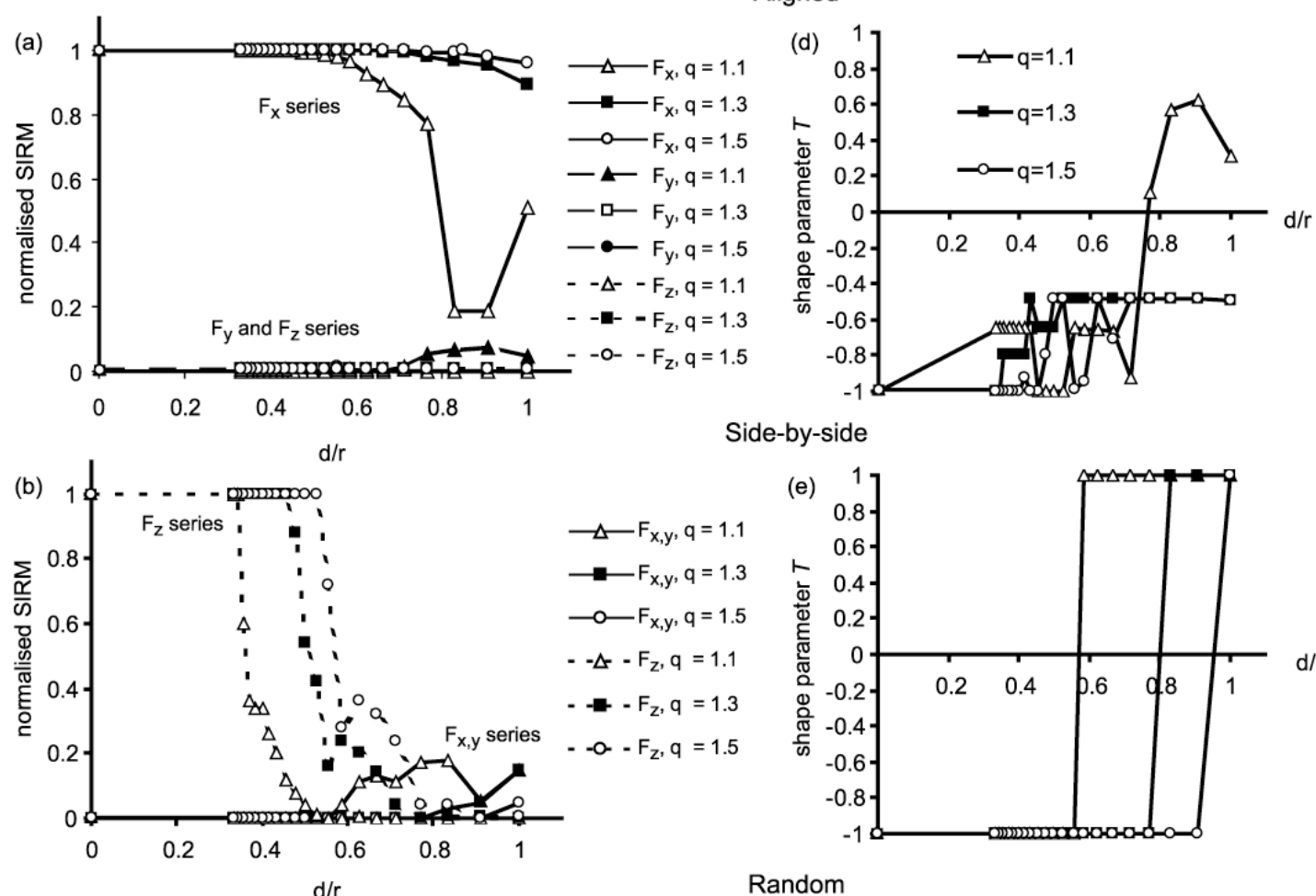

Side-by-side
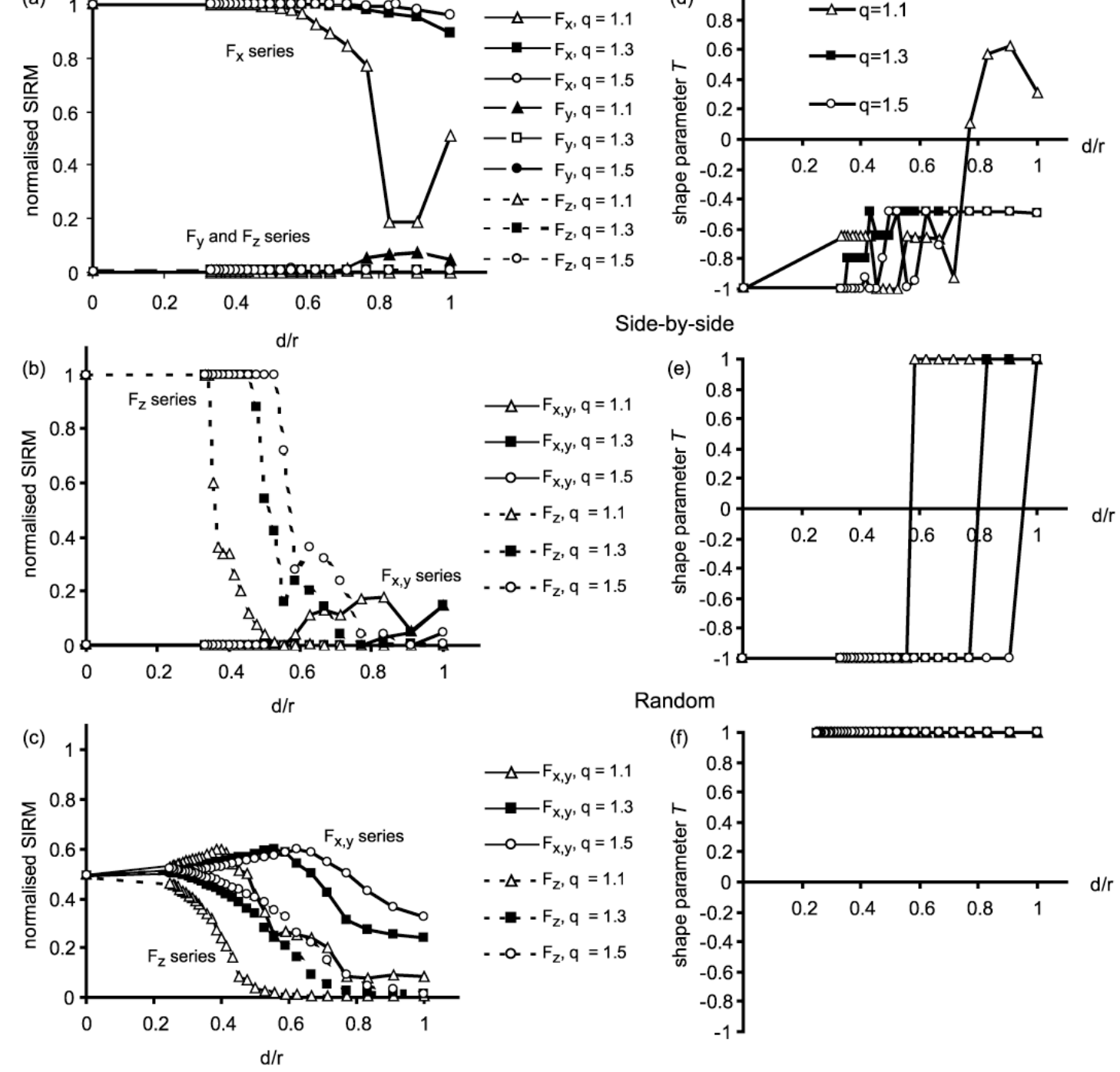

Random

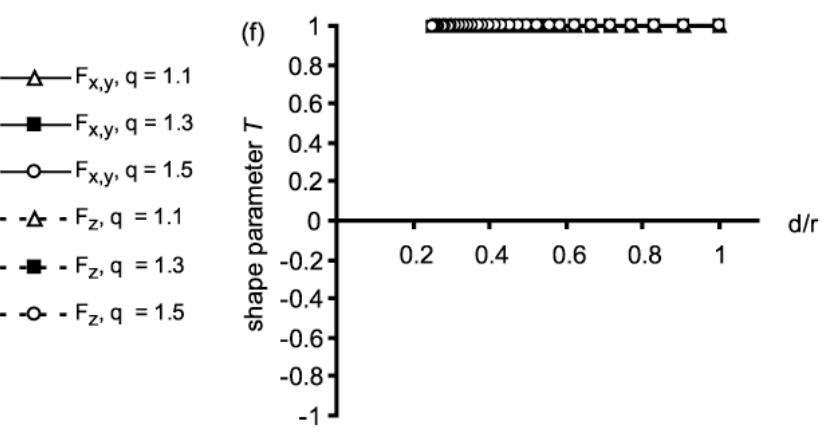

Figure 6. 


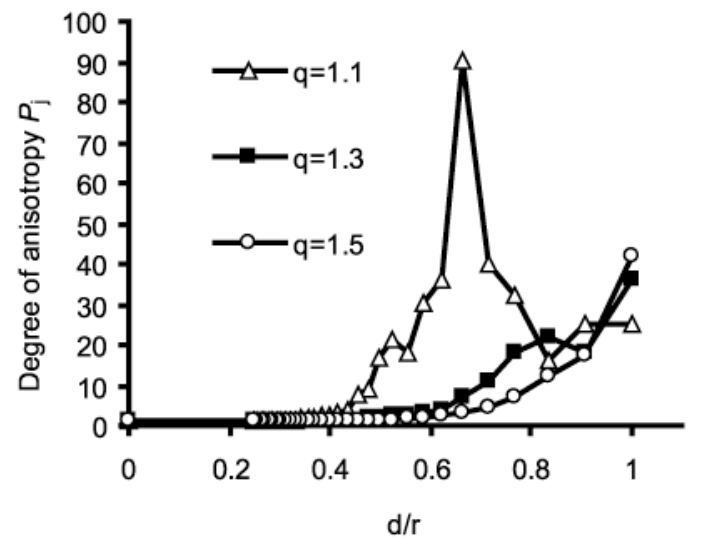

Figure 7.

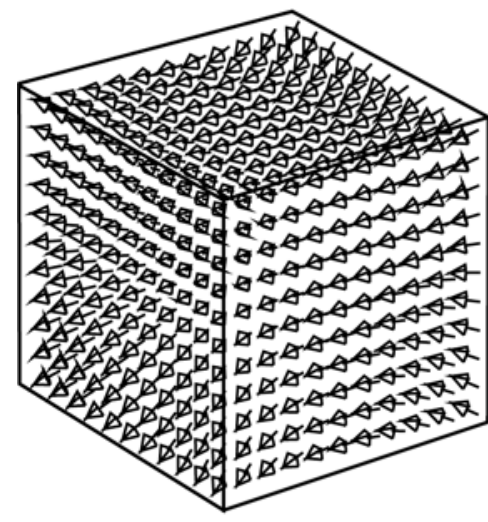

a) single domain (flower state)

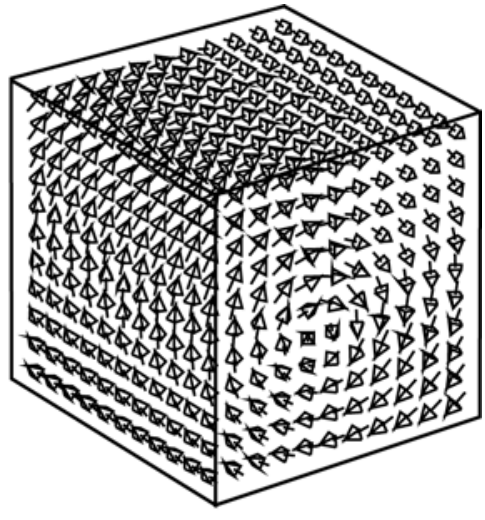

b) vortex state

Figure 8. 


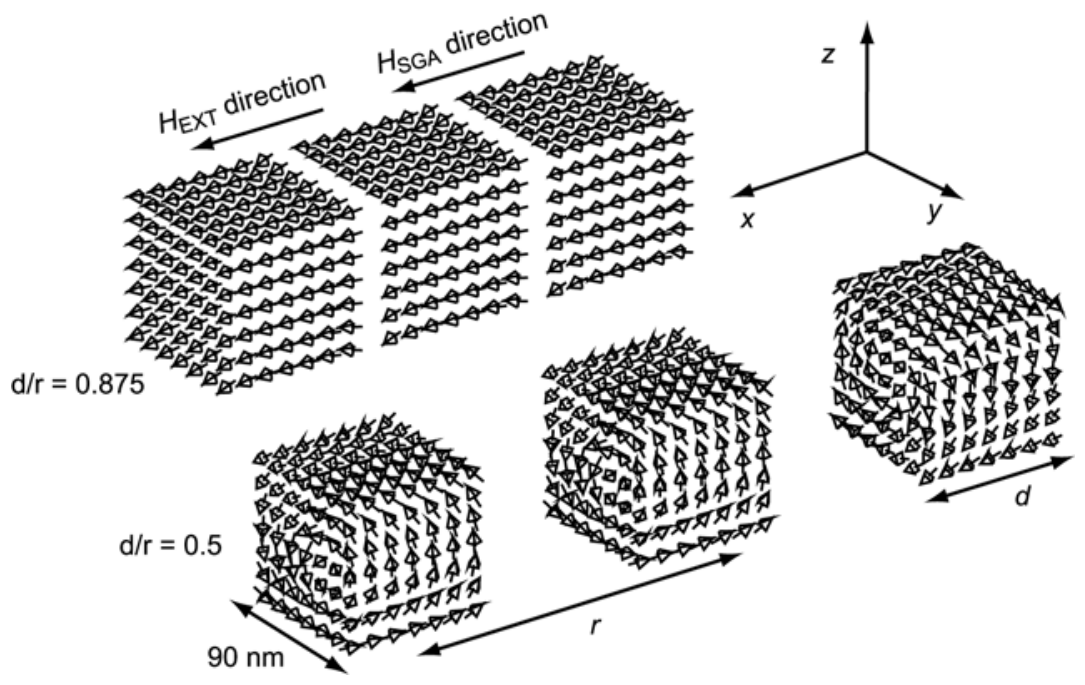

Figure 9.
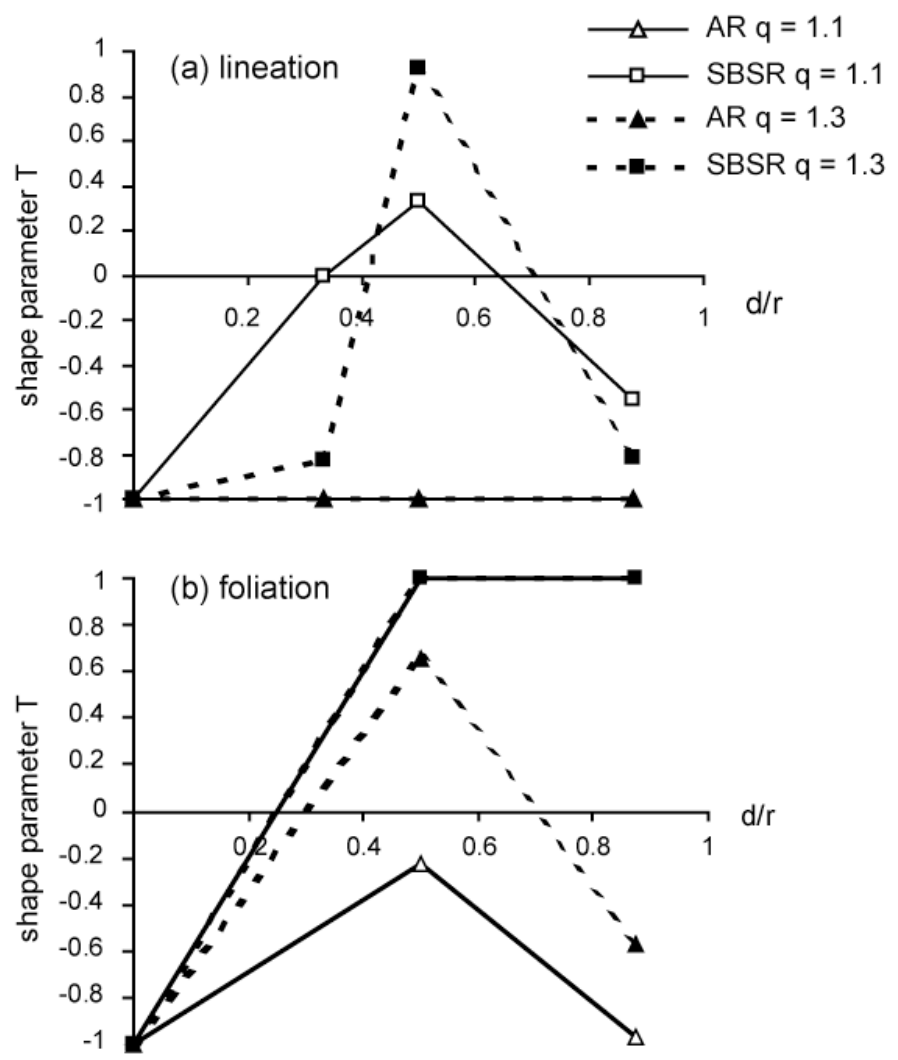

Figure 10. 